\title{
Síntesis óptima de un mecanismo de cinco barras de 2-GDL utilizando técnicas de inteligencia artificial
}

\author{
Maria Bárbara Calva Yáñez ${ }^{1}$, Paola Andrea Niño Suárez ${ }^{1}$, Jorge Alexander Aponte \\ Rodríguez $^{3}$, Eric Santiago Valentín ${ }^{2}$, Edgar Alfredo Portilla Flores ${ }^{2}$, \\ Gabriel Sepúlveda Cervantes ${ }^{2}$ \\ ${ }^{1}$ Instituto Politécnico Nacional, Escuela Superior de Ingeniería Mecánica y Eléctrica \\ Azcapotzalco (SEPI-ESIME-AZC), Cd. de México, \\ México \\ ${ }^{2}$ Instituto Politécnico Nacional, Centro de Innovación y Desarrollo Tecnológico en Cómputo, \\ Cd. de México, México \\ ${ }^{3}$ Universidad Militar Nueva Granada, \\ Bogotá, Colombia \\ b_calva@hotmail.com,jorge.aponte@unimilitar.edu.co, e.santiag.valentin@gmail.com, \\ \{aportilla, pninos, gsepulvedac\}@ipn.mx
}

Resumen. En este trabajo se presenta la síntesis dimensional óptima de un mecanismo de cinco barras para minimizar la fuerza en el eslabón de salida y asegurar el seguimiento de trayectorias en un espacio de trabajo predeterminado. Dicha síntesis se lleva a cabo al proponer un problema de optimización numérica con restricciones considerando aspectos estructurales y de fuerzas del mecanismo en estudio. El algoritmo denominado Evolución Diferencial (ED) es utilizado para resolver el problema de optimización resultante. Un conjunto de 30 ejecuciones independientes del algoritmo se lleva a cabo y el mejor de los resultados alcanzados es comparado con otras posibles soluciones. Se demuestra que el mecanismo óptimo tiene un mejor funcionamiento de acuerdo a la función objetivo seleccionado. Así mismo, se muestran resultados estadísticos paramétricos del rendimiento del algoritmo de ED, los cuales permiten sugerir un alto rendimiento del mismo para resolver problemas de diseño en ingeniería.

Palabras clave: Optimización, mecanismo plano, evolución diferencial.

\section{Optimal Synthesis of a 2-DOF Five Bar Mechanism Using Artificial Intelligence Techniques}

\begin{abstract}
This paper presents the optimal dimensional synthesis of a five -bar mechanism in order to minimize the force on the output link and ensure paths inside an established workspace. The synthesis is carried out by proposing a numerical optimization problem with constrains taking into account structural and force mechanism considerations. The Differential Evolution algorithm is
\end{abstract}


used to solve the resulting optimization problem. A set of 30 independent executions of the algorithm is carried out and the best of the results achieved compared with other possible solutions. It is shown that the optimal mechanism has a better performance according to the selected objective function. In addition, parametric statistical results of the DE algorithm performance are shown; such results suggest a high performance of the DE algorithm for solving engineering design problems.

Keywords: optimization, planar mechanism, differential evolution.

\section{Introducción}

La necesidad de incrementar el rendimiento de procesos industriales con la reducción de consumos de energía y/o recursos ha motivado a los expertos en computación a desarrollar algoritmos para resolver problemas duros de ingeniería, en razón a la alta complejidad que en muchos casos estos involucran. Es así como el planteamiento de problemas de optimización se ha convertido en una alternativa para solucionar estos problemas, permitiendo simplicidad de implementación, flexibilidad de aplicación y capacidad para encontrar buenas respuestas [1].

Los mecanismos planos han sido utilizados en forma extensiva para la solución de diversos problemas en la industria, como es la transmisión de movimiento. La necesidad de ajustar las trayectorias descritas por estos ha derivado en el uso de diferentes metodologías para la síntesis cinemática como son generación de función, trayectoria y movimientos [2]. La primera correlaciona la entrada con la salida del sistema, el segundo busca el control de un punto en el plano de modo que siga alguna trayectoria prescrita, en el tercero se realiza el control de una línea en el plano cuando esta asume algún conjunto de posiciones prescritas. Existen diferentes trabajos relacionados con la síntesis de mecanismos en los cuales a partir de la cinemática del sistema se plantean problemas de optimización numérica para obtener un conjunto de parámetros que describan el sistema, logrando que este se ajuste a un comportamiento requerido en aspectos como el seguimiento de trayectorias o minimización de fuerzas.

En [1] se realiza la síntesis dimensional óptima de un mecanismo de cuatro barras para garantizar la mayor transferencia de fuerza al eslabón de salida. En [3], el diseño de un efector final tipo pinza es realizado a partir de proponer un problema de optimización numérico con el objetivo de mantener la fuerza constante en todo el espacio de trabajo, dicho problema es resuelto utilizando una algoritmo de inteligencia colectiva. En [4] se realiza el diseño óptimo de un intercambiador de calor utilizando firefly como algoritmo. Los autores de [5] realizan la síntesis óptima para el seguimiento de trayectoria en mecanismos de cuatro barras utilizando programación cuadrática secuencial (SQP). Para el diseño de un efector final en el cual se mantiene la fuerza constante en el espacio de trabajo, [6] utiliza el algoritmo de Evolución Diferencial.

En diversas aplicaciones de ingeniería se requiere accionar mecanismos con el menor consumo de energía posible y con alta precisión en el posicionamiento, como son los sistemas automáticos de soldadura, corte o ensamble. En este trabajo se presenta la síntesis dimensional de un mecanismo de cinco barras en el cual además de lograr minimizar la fuerza en el eslabón de salida considerando aspectos dinámicos del 
Síntesis óptima de un mecanismo de cinco barras de 2-GDL utilizando técnicas de inteligencia ...

sistema, se ajusta el seguimiento de una trayectoria en un espacio de trabajo predeterminado, permitiendo ejecutar tareas como las ya mencionadas, aportando un enfoque en el cual se garantiza la menor fuerza en el actuador de salida para cualquier trayectoria contenida en el espacio de trabajo.

La organización del artículo es la siguiente: la Sección 2 describe el problema de síntesis de mecanismos, con una breve explicación de la cinemática, así como el análisis de fuerzas. En la Sección 3 se presentan las estrategias de optimización, analizando a la función objetivo y a las restricciones de diseño, además se presenta el planteamiento del problema de optimización. Posteriormente, en la Sección la Sección 4 se muestra el algoritmo empleado y algunos aspectos de la implementación computacional. Finalmente, en la Sección 5 se presentan y discuten los resultados obtenidos, y en la Sección 6 se exponen las conclusiones correspondientes.

\section{Cinemática del mecanismo}

El seguimiento de trayectorias en el plano ha sido solucionado con el uso de diferentes mecanismos, a continuación se presenta la síntesis de un mecanismo de cinco barras que minimiza la fuerza horizontal generada sobre el eslabón 5 y además facilita el ajuste paramétrico de trayectorias en un espacio de trabajo predeterminado.

\subsection{Síntesis cinemática}

El mecanismo mostrado en la Fig. 1, está compuesto por la barra 1 fija, mientras que la barra 2 realiza un movimiento de rotación pura. La barra 3 sirve de acoplador entre las deslizaderas 4 con movimiento de rotación y traslación (con la barra 2), y 5 la cual se traslada sobre la barra fija, siendo este el elemento de salida de la cadena cinemática. El punto de unión entre las barras 3 y 4 puede describir trayectorias en el plano.

Utilizando el criterio de Grubler - Kutzbach se determina la movilidad $(m)$ del mecanismo (ecuación 1).

$$
m=3(n-1)-2 J_{1}-J_{2},
$$

dónde:

$n=$ número de eslabones

$J_{1}=$ pares de un grado de libertad

$J_{2}=$ pares de dos grado de libertad

Para este mecanismo:

$$
m=3(5-1)-2(5)-0=2 .
$$

Los resultados de la ecuación (2) indican que este mecanismo requiere dos movimientos independientes para que pueda realizar una trayectoria previamente establecida. Para realizar el análisis cinemático del mecanismo, este puede ser representado con el diagrama esquemático mostrado en la Fig. 2. 


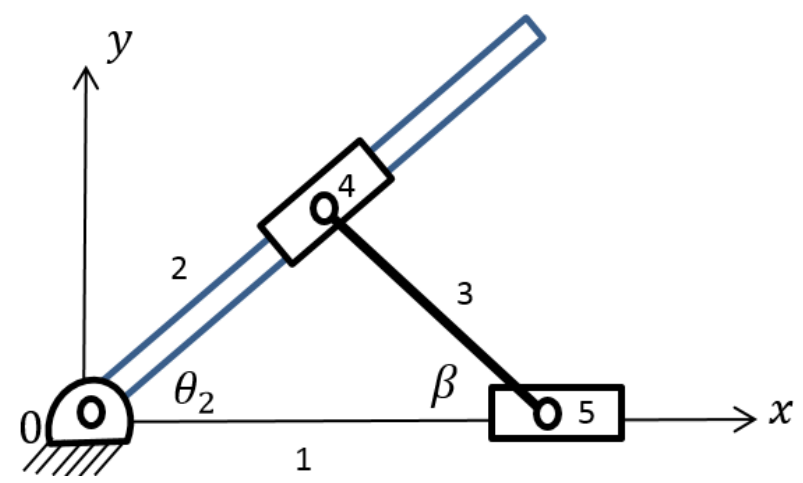

Fig. 1. Esquema general del mecanismo.

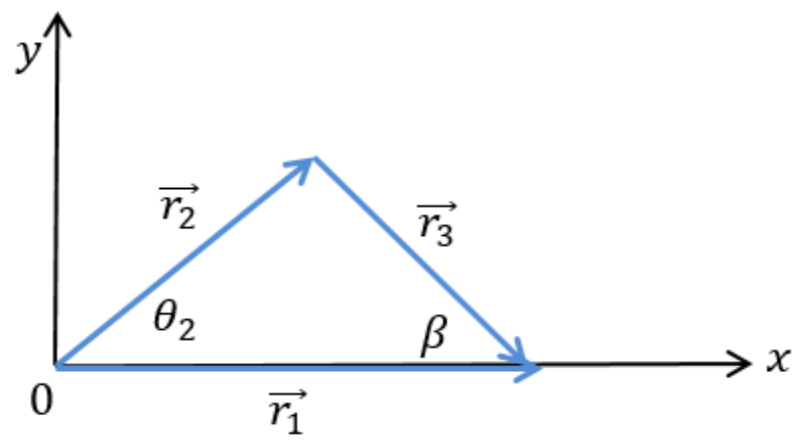

Fig. 2. Esquemático del mecanismo.

Se tendrá en cuenta que los vectores $\overrightarrow{r_{1}} y \overrightarrow{r_{2}}$ cambian en magnitud, $\overrightarrow{r_{3}}$ permanece constante en magnitud y los ángulos $\theta_{2}$ y $\beta$ cambian, mientras que el eslabón 1 no cambia en dirección $\left(\theta_{1}=0\right.$ constante $)$. Para los análisis a continuación se hará $180-\beta=\theta_{3}$

La ecuación de cierre del circuito es:

$$
\overrightarrow{r_{1}}=\overrightarrow{r_{2}}+\overrightarrow{r_{3}}
$$

Puesta en forma polar:

$$
r_{1} e^{j \theta_{1}}=r_{2} e^{j \theta_{2}}+r_{3} e^{j \theta_{3}}
$$

Utilizando Euler:

$$
r_{1}\left(\cos \theta_{1}+j \operatorname{sen} \theta_{1}\right)=r_{2}\left(\cos \theta_{2}+j \operatorname{sen} \theta_{2}\right)+r_{3}\left(\cos \theta_{3}+j \operatorname{sen} \theta_{3}\right) .
$$

Separando la parte real e imaginaria de (5) y aplicando las condiciones de funcionamiento del mecanismo, se obtiene:

$$
\begin{gathered}
r_{1}=r_{2}\left(\cos \theta_{2}\right)+r_{3}\left(\cos \theta_{3}\right), \\
\theta_{3}=-\operatorname{arcsen}\left(\left(r_{2} \operatorname{sen} \theta_{2}\right) / r_{3}\right) .
\end{gathered}
$$




\subsection{Análisis de fuerzas}

Para el análisis de fuerzas se consideró el sistema en equilibrio, con una fuerza $\mathrm{P}$, aplicada al eslabón 4. En la Fig. 3 se muestra el diagrama de cuerpo libre para este elemento.

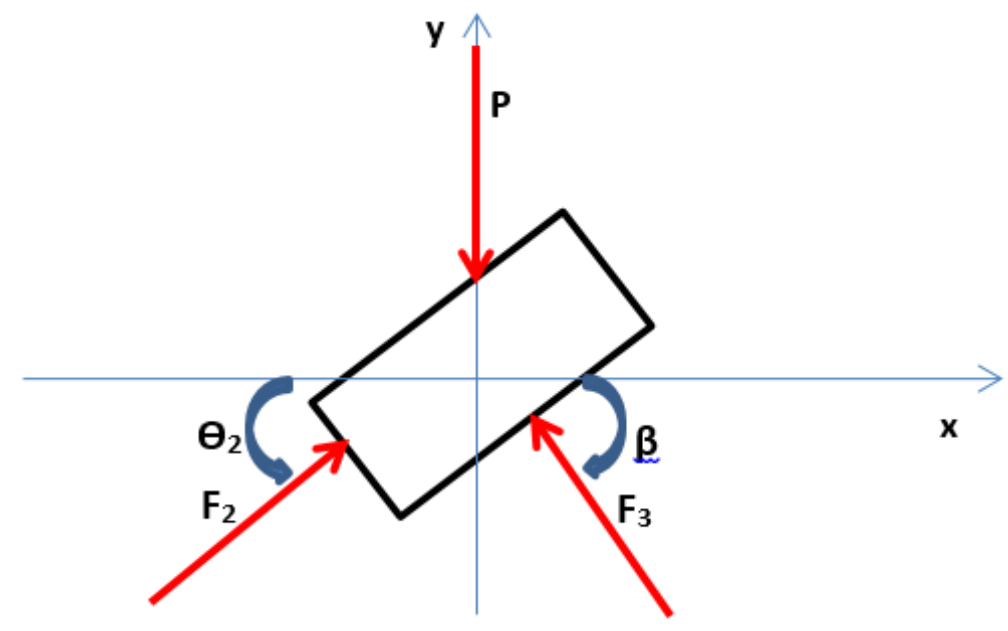

Fig. 3. Diagrama de cuerpo libre del eslabón 4.

En la Figura 3:

$\mathrm{F}_{3}=$ fuerza ejercida por el eslabón 3 sobre 4,

$\mathrm{F}_{2}=$ fuerza ejercida por el eslabón 2 sobre 4 ,

$\mathrm{P}=$ fuerza.

Con base en las leyes de Newton se plantean las ecuaciones de equilibrio:

$$
\begin{gathered}
\sum F_{X}=0 \quad F_{2} \cos \theta_{2}-F_{3} \cos \beta=0, \\
\sum F_{Y}=0 \quad F_{2} \operatorname{sen} \theta_{2}+F_{3} \operatorname{sen} \beta-P=0 .
\end{gathered}
$$

Resolviendo se llega a:

$$
F_{3}=\frac{P}{\operatorname{sen} \beta+\tan \theta_{2} \cos \beta},
$$

siendo

$$
\beta=\frac{r_{2}}{r_{3}} \sin \theta_{2}
$$

Para reducir los esfuerzos sobre el elemento 3 y minimizar la fuerza que se requiere al utilizar un accionamiento horizontal sobre el eslabón 5, se procede a elaborar el diagrama de cuerpo libre de este, ilustrado en la Fig. 4. 


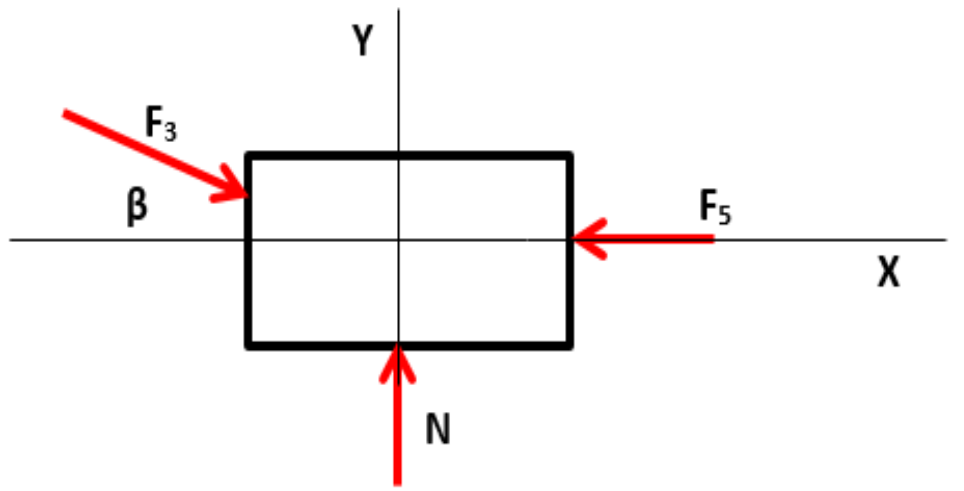

Fig. 4. Diagrama de cuerpo libre del eslabón 5.

Aplicando las condiciones de equilibrio se llega a:

$$
\begin{aligned}
& \sum F_{X}=0-F_{5}+F_{3} \cos \beta=0 \Rightarrow F_{5}=F_{3} \cos \beta \\
& \sum F_{Y}=0 \quad N-F_{3} \operatorname{sen} \beta=0 \Rightarrow N=F_{3} \operatorname{sen} \beta .
\end{aligned}
$$

Sustituyendo (10) en (12) y despejando se encuentra que la fuerza sobre el eslabón 5 está dada por

$$
F_{5}=\frac{P}{\tan \beta+\tan \theta_{2}}
$$

\section{Planteamiento del problema de optimización}

Una vez realizados el análisis cinemático y de fuerzas, el diseño del mecanismo se plantea como un problema de optimización numérico, en el cual se deben especificar las relaciones matemáticas que permitan evaluar el desempeño del mismo.

\subsection{Función objetivo}

Como ya se planteó previamente, en este trabajo se desea determinar el tamaño de los eslabones que permitan seguir una trayectoria en un espacio de trabajo determinado por los puntos $\Omega$ y que además minimice la fuerza transmitida al eslabón de salida, siendo $\Omega=\{(10,10),(80,10),(80,50),(10,50)\}$, como se ilustra en la Fig. 5 .

Considerando las ecuaciones obtenidas en el análisis de fuerzas, se plantea el problema de optimización numérica mono objetivo descrito por (14), para obtener la solución al problema de diseño para minimizar la fuerza sobre el eslabón 5:

$$
\mathrm{f}\left(\beta_{i}, \theta_{2}^{i}\right)=\sum_{i=1}^{n} \frac{P}{\operatorname{sen} \beta_{i}+\tan \theta_{2}^{i} \cos \beta_{i}} .
$$




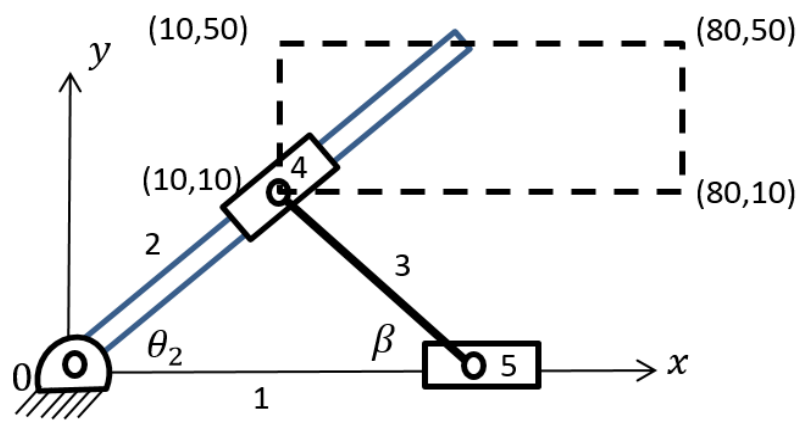

Fig. 5. Trayectoria a realizar por el punto de conexión entre los eslabones 4 y 3 .

\subsection{Restricciones de diseño}

El cumplimiento de condiciones de funcionamiento está determinada por la dimensionalidad del mecanismo, el cual se establece como un conjunto de restricciones en el problema de optimización.

Para garantizar que el eslabón 4 pase por los puntos de precisión y que además $\beta \neq$ $90^{\circ}$ (evitando los problemas de montaje y funcionamiento que se generan cuando $\beta=$ $\left.90^{\circ}\right)$, se establece que $r_{3}>r_{2}^{i} \operatorname{sen} \theta_{2}^{i}$. Por otro lado al establecer que $r_{3} \operatorname{sen} \beta_{i}=y_{i}$, con $i=1 \ldots 4$, se garantiza el paso del eslabón 3 por los puntos de precisión $\Omega$

\subsection{Vector de variables de diseño}

Sea el vector de variables de diseño para el mecanismo de cinco barras establecido como:

$$
\vec{x}=\left[r_{3}, \beta_{1}, \beta_{2}, \beta_{3}, \beta_{4}\right]^{T}=\left[x_{1}, x_{2}, x_{3}, x_{4}, x_{5}\right]^{T},
$$

donde $r_{3}$ es la longitud de la barra acopladora y $\beta_{1}, \beta_{2}, \beta_{3}, \beta_{4}$ son los ángulos que forma el eslabón 5 con respecto a la barra fija.

\subsection{Problema de optimización}

Teniendo en cuenta lo planteado en la sección 3.2, el problema de optimización numérica queda descrito por las ecuaciones 17 a 29.

$$
\min _{x \in \mathbb{R}^{5}} \sum_{i=1}^{n} \frac{P}{\operatorname{sen} x_{2}+\tan \theta_{2}^{i} \cos x_{2}} \mathrm{n} \in \mathbb{R}^{5},
$$

sujeto a:

$$
\begin{aligned}
& g_{1}(\vec{x})=r_{2}^{1} \operatorname{sen} \theta_{2}^{1}-x_{1} \leq 0 \\
& g_{2}(\vec{x})=r_{2}^{2} \operatorname{sen} \theta_{2}^{2}-x_{1} \leq 0 \\
& g_{3}(\vec{x})=r_{2}^{3} \operatorname{sen} \theta_{2}^{3}-x_{1} \leq 0
\end{aligned}
$$




$$
\begin{aligned}
& g_{4}(\vec{x})=r_{2}^{4} \operatorname{sen} \theta_{2}^{4}-x_{1} \leq 0 \\
& h_{1}(\vec{x})=x_{1} \operatorname{sen} x_{2}-Y_{y}^{1}=0 \\
& h_{2}(\vec{x})=x_{1} \operatorname{sen} x_{3}-Y_{y}^{2}=0 \\
& h_{3}(\vec{x})=x_{1} \operatorname{sen} x_{4}-Y_{y}^{3}=0 \\
& h_{4}(\vec{x})=x_{1} \operatorname{sen} x_{5}-Y_{y}^{4}=0 .
\end{aligned}
$$

Con cotas de:

$$
\begin{aligned}
0 & <x_{1}<110 \\
0<x_{i} & <\frac{\pi}{2} \quad i=2, \ldots, 5,
\end{aligned}
$$

donde:

$$
\begin{gathered}
r_{2}^{i}=\sqrt{\left(x_{i}\right)^{2}+\left(y_{i}\right)^{2},} \\
\theta_{2}^{i}=\tan ^{-1}\left(\frac{y_{i}}{x_{i}}\right),
\end{gathered}
$$

siendo $\mathrm{P}=80 \mathrm{~kg}$, y $y_{i}$ la ordenada de los puntos de precisión $\Omega$.

\section{Algoritmo de optimización}

El algoritmo de Evolución Diferencial (ED) es una de las heurísticas más utilizadas dentro de la computación evolutiva, ya que resuelve de manera eficiente los problemas duros de ingeniería [7]. ED parte de una población inicial de soluciones aleatorias y en cada generación se producen individuos de prueba aplicando operadores de cruza y mutación. La aptitud de cada nuevo individuo se evalúa compitiendo con un individuo de la población actual y así determina cuál de ellos se conservará para la siguiente generación. Las ventajas de que presenta ED, es el número reducido de parámetros de control tales como el tamaño de población (NP), el factor de cruza (CR) y el factor de mutación $(\mathrm{F})$. Las características principales características de esta herramienta son:

- Representación de individuos,

- Selección de padres,

- Recombinación o cruza,

- Mutación y selección de sobrevivientes y variantes.

El diagrama de bloques correspondiente al algoritmo evolución diferencial se muestra en la figura 6.

Para el manejo de las restricciones se utilizan las reglas de DEB [8].

1. Entre dos individuos factibles, se escoge al que tenga la mejor función objetivo,

2. Entre un individuo factible y otro no factible, se escoge al factible,

3. Entre dos individuos no factibles, se escoge al que tenga un valor menor en la suma de violación a las restricciones. 


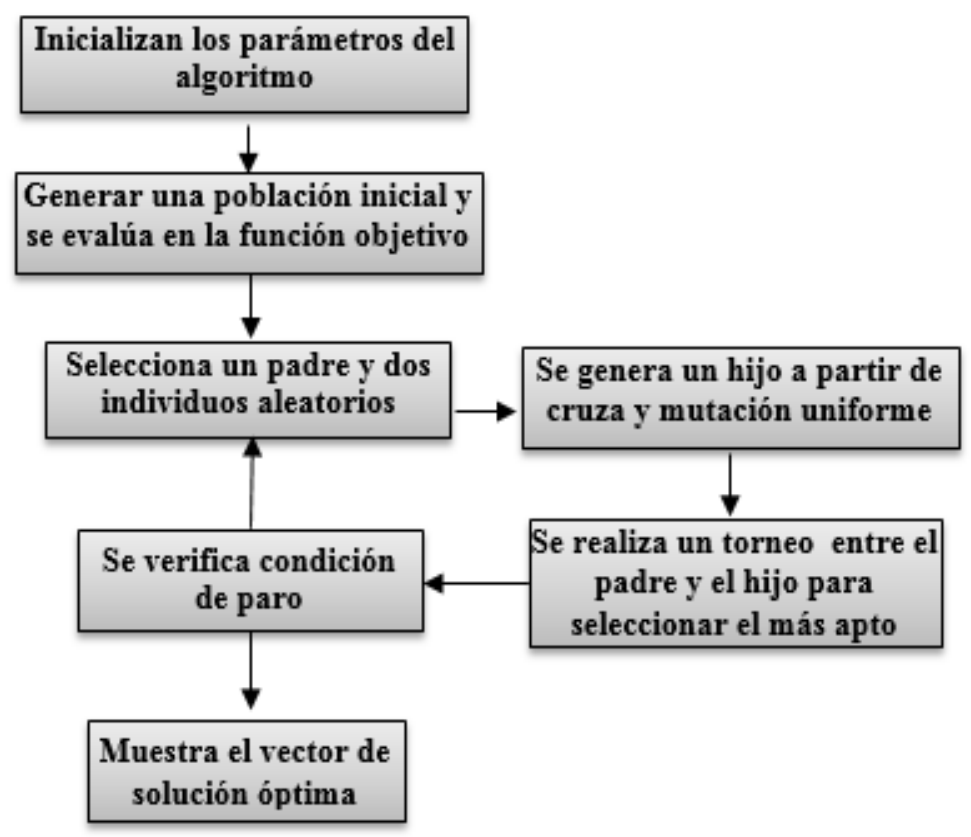

Fig. 6. Diagrama de bloques del algoritmo ED.

\subsection{Implementación computacional}

La implementación del algoritmo se realizó en Matlab R2013b®, ejecutado en una computadora con 8GB RAM, procesador INTEL CORE i7 a 2.2 GB y un sistema operativo Microsoft Windows 8 . El programa se implementa en un módulo donde se calcula el valor de la función objetivo y las restricciones. Para el manejo de restricciones se realizan una sumatoria de violación de estas.

En la Tabla 1 se muestran las características del problema $N$ es el número de variables del problema, li es el número de restricciones de desigualdad lineales, ni son las restricciones de desigualdad no lineales, le restricciones de igualdad lineales, ne las restricciones de igualdad no lineales, y $\boldsymbol{\rho}$ que es la complejidad del problema [9].

Tabla 1. Características del problema.

\begin{tabular}{llllll}
\hline & & & & $\rho$ \\
\hline 5 & 4 & 4 & 0 & 0 & 0.0 \\
& & & & 00 \\
& & & & $\%$ \\
\hline
\end{tabular}

El parámetro $\rho$ mide la complejidad del problema, el cual es la relación entre la zona factible y el espacio de búsqueda, definido por Michalewicz y Shoenauer [10] como $\rho=|\mathrm{F}| /|\mathrm{S}|$, donde $|\mathrm{S}|$ es el número total de soluciones generadas aleatoriamente y $|\mathrm{F}|$ es el número de soluciones factibles obtenidas a partir de las soluciones generadas 
Maria Bárbara Calva Yáñez, Paola Andrea Niño Suárez, Jorge Alexander Aponte Rodríguez, et al.

aleatoriamente. Cuanto más disminuye $\rho$ la complejidad para encontrar soluciones aumenta, representando un mayor reto para los algoritmos.

Para encontrar el valor del parámetro $\rho$ de este caso de estudio se generan 1 millón de soluciones aleatorias, dentro de las cotas mencionadas, cabe resaltar que el problema tiene restricciones de igualdad y haciendo necesario el uso de un $\varepsilon=0.0001$.

\section{Resultados}

Se realizaron un conjunto de treinta simulaciones independientes con los siguientes parámetros: Un tamaño de población $N P=50$, número máximo de generaciones $G M A X$ $=4,999$ con un numero de evaluaciones $E V A L=250,000$ factor de cruza $C R=[0.8$, $1.0]$ por ejecución, y factor de escala $F=[0.3,0.9]$ por corrida. Los resultados obtenidos se muestran en la Tabla 2.

Tabla 2. Resultados de las 30 simulaciones.

\begin{tabular}{ccccccc}
\hline Sim & $\mathbf{r}_{\mathbf{3}}$ & $\boldsymbol{\beta}_{\mathbf{1}}$ & $\boldsymbol{\beta}_{\mathbf{2}}$ & $\boldsymbol{\beta}_{\mathbf{3}}$ & $\boldsymbol{\beta}_{\mathbf{4}}$ & $\boldsymbol{F} \boldsymbol{O}$ \\
\hline 1 & 53.4658913 & 0.18814504 & 0.18814504 & 1.20874940 & 1.20874940 & 425.351273 \\
2 & 53.4658914 & 0.18814504 & 0.18814504 & 1.20874940 & 1.20874940 & 425.351273 \\
3 & 53.4658914 & 0.18814504 & 0.18814504 & 1.20874939 & 1.20874939 & 425.351273 \\
4 & 53.4658915 & 0.18814504 & 0.18814504 & 1.20874939 & 1.20874939 & 425.351273 \\
5 & 53.4658914 & 0.18814504 & 0.18814504 & 1.20874940 & 1.20874940 & 425.351273 \\
6 & 53.4658913 & 0.18814504 & 0.18814504 & 1.20874940 & 1.20874940 & 425.351273 \\
7 & 53.4658914 & 0.18814504 & 0.18814504 & 1.20874939 & 1.20874939 & 425.351273 \\
8 & 53.4658914 & 0.18814504 & 0.18814504 & 1.20874940 & 1.20874940 & 425.351273 \\
9 & 53.4658915 & 0.18814504 & 0.18814504 & 1.20874939 & 1.20874939 & 425.351273 \\
10 & 53.4658914 & 0.18814504 & 0.18814504 & 1.20874940 & 1.20874940 & 425.351273 \\
11 & 53.4658914 & 0.18814504 & 0.18814504 & 1.20874940 & 1.20874940 & 425.351273 \\
12 & 53.4658914 & 0.18814504 & 0.18814504 & 1.20874939 & 1.20874939 & 425.351273 \\
13 & 53.4658915 & 0.18814504 & 0.18814504 & 1.20874939 & 1.20874939 & 425.351273 \\
14 & 53.4658913 & 0.18814504 & 0.18814504 & 1.20874940 & 1.20874940 & 425.351273 \\
15 & 53.4658914 & 0.18814504 & 0.18814504 & 1.20874939 & 1.20874939 & 425.351273 \\
16 & 53.4658914 & 0.18814504 & 0.18814504 & 1.20874939 & 1.20874939 & 425.351273 \\
17 & 53.4658914 & 0.18814504 & 0.18814504 & 1.20874940 & 1.20874940 & 425.351273 \\
18 & 53.4658914 & 0.18814504 & 0.18814504 & 1.20874940 & 1.20874940 & 425.351273 \\
19 & 53.4658043 & 0.18814535 & 0.18814535 & 1.20875370 & 1.20875370 & 425.351273 \\
20 & 53.2848938 & 0.18879087 & 0.18879142 & 1.21783176 & 1.21783536 & 425.370477 \\
21 & 52.8198721 & 0.19047300 & 0.19047078 & 1.24256183 & 1.24256582 & 425.613713 \\
22 & 52.8117989 & 0.19050330 & 0.19050192 & 1.24301109 & 1.24301236 & 425.619245 \\
23 & 52.7549530 & 0.19071135 & 0.19070925 & 1.24619292 & 1.24619425 & 425.672008 \\
24 & 52.7226199 & 0.19082724 & 0.19082669 & 1.24802558 & 1.24802304 & 425.705390 \\
25 & 52.6434549 & 0.19111771 & 0.19111900 & 1.25255429 & 1.25254580 & 425.790906 \\
26 & 52.5926037 & 0.19130702 & 0.19130610 & 1.25549575 & 1.25549232 & 425.852691 \\
\hline & & & & & & \\
\end{tabular}


Síntesis óptima de un mecanismo de cinco barras de 2-GDL utilizando técnicas de inteligencia ...

\begin{tabular}{ccccccc}
\hline Sim & $\mathbf{r}_{\mathbf{3}}$ & $\boldsymbol{\beta}_{\mathbf{1}}$ & $\boldsymbol{\beta}_{\mathbf{2}}$ & $\boldsymbol{\beta}_{\mathbf{3}}$ & $\boldsymbol{\beta}_{\mathbf{4}}$ & $\boldsymbol{F O}$ \\
\hline 27 & 52.5121977 & 0.19160244 & 0.19160282 & 1.26022465 & 1.26022310 & 425.961349 \\
28 & 52.1208640 & 0.19305699 & 0.19305955 & 1.28453759 & 1.28454012 & 426.699432 \\
29 & 51.9761886 & 0.19360197 & 0.19360344 & 1.29415343 & 1.29415080 & 427.077478 \\
30 & 51.6893145 & 0.19468957 & 0.19469123 & 1.31442892 & 1.31442442 & 428.044903 \\
\hline
\end{tabular}

En la Tabla 2 se muestran los mejores vectores de solución obtenidos en cada simulación, ordenadas de manera ascendente, considerando el valor de función objetivo. Como se puede observar en las primeras 19 simulaciones se encuentra la misma solución, lo cual se debe la sintonización de parámetros del algoritmo que se realizó. En las 11 simulaciones restantes, se encuentran respuestas diferentes en razón a la alta complejidad del problema según el $\rho$ calculado, lo cual repercute en el valor de la desviación estándar (0.6104) presentada en la Tabla 3.

Tabla 3. Estadísticas paramétricas.

\begin{tabular}{cc}
\hline Mejor & 425.351272969054 \\
Mediana & 425.351272969054 \\
Peor & 428.044903376276 \\
Promedio & 425.636059394308 \\
Desviación estándar & 0.610428900392 \\
\hline
\end{tabular}

En la Fig. 7a se muestra el comportamiento de los individuos que entran a la zona factible, donde se ilustran tres casos distintos, destacándose que antes de las 15,000 evaluaciones (300 generaciones) todos los ínvidos se encuentran en la zona factible, demostrando un buen desempeño del algoritmo. En la Fig. 7b, se realiza el análisis de convergencia para los tres casos representados anteriormente, encontrando que el caso promedio requiere menos evaluaciones para lograr la convergencia, mientras que el peor caso, no logra un mejor resultado aunque su población entró en menos evaluaciones a la zona factible

Las dimensiones obtenidas al solucionar el problema de optimización planteado, deben ser factibles y desde el punto de vista de ingeniería mecatrónica, de fácil manufactura y ensamble, lo cual se logra a partir de la mejor solución encontrada hasta el momento, con los resultados presentados en la Tabla 4 e ilustrados en la figura 8. La fuerza en cada una de las cuatro posiciones para el eslabón 3 también se especifica en dicha tabla, destacándose el mayor valor en la posición 2, a consecuencia de tener los eslabones con un ángulo pequeño con respecto a la horizontal.

Es posible proponer otras alternativas basadas en la experiencia del diseñador para el seguimiento de la trayectoria sin plantear un problema de optimización. De esta manera se puede considerar colocar al eslabón 3 a $90^{\circ}$ del eslabón fijo mientras hace el recorrido en el espacio de trabajo de los puntos 3 a 4 , lo cual hace que la sumatoria total de fuerzas transmitida a la deslizadera 5 sea de mayor que la obtenida al solucionar el problema de optimización planteado. Con un razonamiento similar se puede considerar una solución en la que la longitud de los eslabones 2 y 3 sean iguales en la posición 3 , buscando una distribución uniforme de carga en dichos eslabones, de igual manera en estas condiciones la sumatoria total de fuerzas a lo largo de la trayectoria es mayor a la 
Maria Bárbara Calva Yáñez, Paola Andrea Niño Suárez, Jorge Alexander Aponte Rodríguez, et al.

mejor solución encontrada hasta el momento mediante el problema de optimización dichos resultados se pueden observar en la Tabla 5.

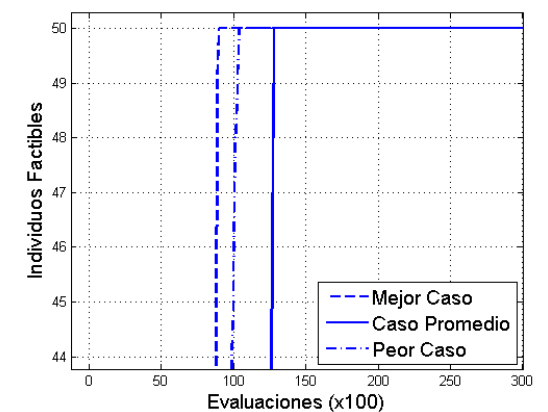

Fig 7a. Individuos factibles por evaluación.

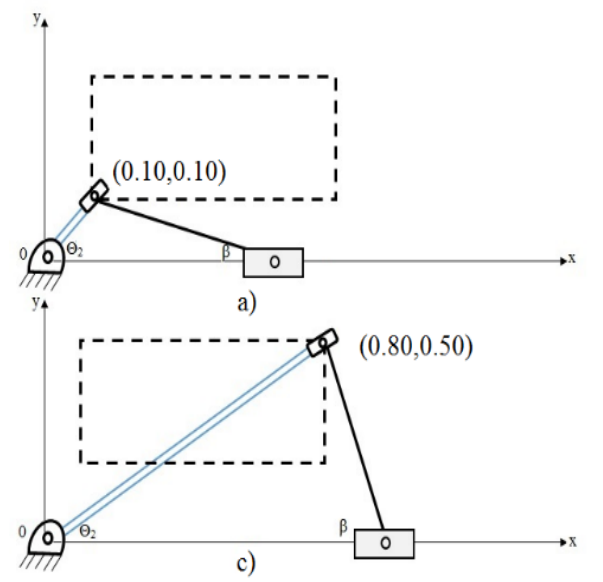

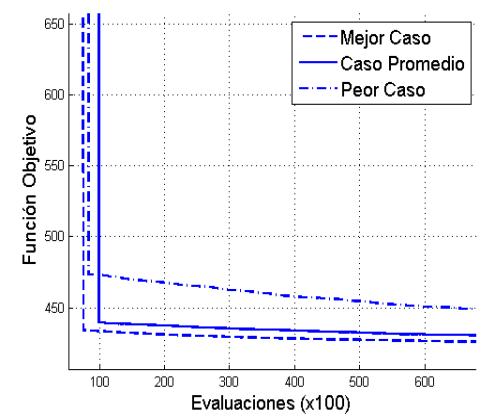

Fig 7b. Convergencia de la función objetivo.

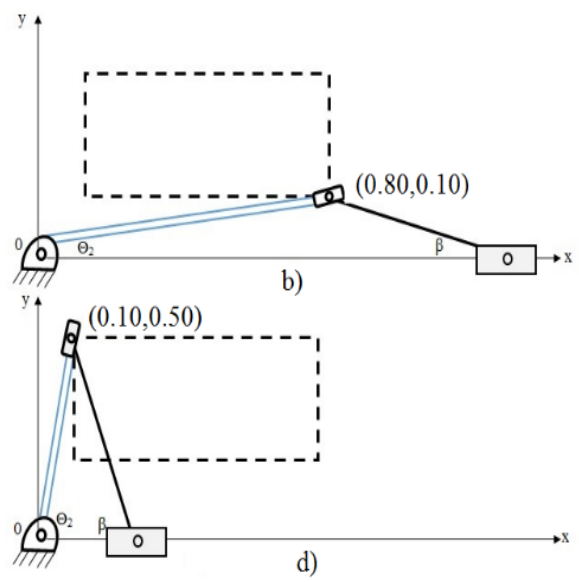

Fig 8. Posiciones alcanzadas por el mecanismo con las dimensiones encontradas.

Tabla 4. Dimensiones de la mejor solución encontrada hasta el momento.

\begin{tabular}{ccccccc}
\hline Posición & $\mathbf{r}_{1}[\mathbf{m}]$ & $\mathbf{r}_{2}[\mathbf{m}]$ & $\mathbf{r}_{3}[\mathbf{m}]$ & $\boldsymbol{\Theta}_{2}[\mathbf{R a d}]$ & $\boldsymbol{\beta}[\mathbf{R a d}]$ & $\mathbf{F}_{3}[\mathbf{K g F}]$ \\
\hline$(0.10,0.10)$ & 0.62757554 & 0.14142135 & 0.53465891 & 0.78539816 & 0.18814504 & 68.411744 \\
$(0.80,0.10)$ & 1.32522392 & 0.80622577 & 0.53465891 & 0.12435499 & 0.18814504 & 258.205195 \\
$(0.80,0.50)$ & 0.99136380 & 0.94339811 & 0.53465891 & 0.55859932 & 1.20874940 & 69.1717183 \\
$(0.10,0.50)$ & 0.46491057 & 0.50990195 & 0.53465891 & 1.37340077 & 1.20874940 & 29.562623 \\
\hline
\end{tabular}

Tabla 5. Sumatoria de Fuerzas para diseños basados en la experiencia.

\begin{tabular}{cccc}
\hline $\mathbf{r}_{2}[\mathbf{m}]$ & $\mathbf{r}_{3}[\mathbf{m}]$ & $\boldsymbol{\beta}[\mathbf{r a d}]$ & $\mathbf{F}_{\mathbf{3}}[\mathbf{K g F}]$ \\
\hline 0.94339811 & 0.53465891 & 1.570793 & 513.5596216 \\
0.50990195 & 0.50990195 & 0.197 & 432.5931608 \\
\hline
\end{tabular}




\section{Conclusiones}

En este artículo se presentó la síntesis de un mecanismo de cinco barras para minimizar la fuerza en el eslabón de salida, asegurando el seguimiento de trayectorias dentro de un espacio de trabajo predeterminado con base en el algoritmo de evolución diferencial. De los resultados obtenidos se puede establecer que los algoritmos heurísticos son una herramienta viable para la solución de problemas duros de ingeniería, ya que permiten la reconfiguración del sistema a diseñar, modificando algunos parámetros del problema en el algoritmo.

La correcta interpretación del problema y la formulación de las restricciones, así como la sintonización de los parámetros del algoritmo requiere especial atención para la obtención de soluciones factibles y físicamente realizables. En los problemas de optimización que involucran restricciones de igualdad el manejo del parámetro $\varepsilon$ influye en la obtención de buenas soluciones, teniendo en cuenta que este genera una margen de error aceptable, según la experiencia de quien resuelve el problema.

Con la solución encontrada se garantiza que la fuerza requerida por un accionamiento horizontal que mueva al eslabón 5, será mínima, logrando además reducir los esfuerzos generados sobre el eslabón acoplador 3. Al realizar un dibujó esquemático se comprueba que las dimensiones establecidas permiten alcanzar los puntos de precisión y que se pueden realizar trayectorias que estén contenidas en el espacio de trabajo que se genera con los cuatro puntos de precisión especificados, como se observa en la Fig. 8.

Agradecimientos. Los autores agradecen el apoyo recibido por el Instituto Politécnico Nacional a través de la Secretaría de Investigación y Posgrado vía los proyecto SIP 20161615, SIP 20161167 así como a los programas de EDI y COFAA. Los tres primeros autores agradecen al Consejo Nacional de Ciencia y Tecnología de México (CONACyT-México) por la beca otorgada para estudios de Posgrado en ESIMEAZCAPOTZALCO-IPN y CIDETEC-IPN, respectivamente. El segundo autor agradece a la Universidad Militar Nueva Granada por la comisión de estudios.

\section{Referencias}

1. Portilla Flores, E.A., Calva Yañez, M.B., Villareal Cervantes, M.G., Niño Suarez, P.A., Sepúlveda, C.G.: An Optimum Synthesis of a Planar Mechanism Using a Dynamic-based Approach. IEEE Latin America Transactions, Vol. 13, No. 5, pp. 1497-1503 (2015)

2. Norton, R.L.: Diseño de Maquinaria. Mc Graw Hill (2014)

3. Santiago Valentín, E.: Optimización de Sistemas Mecatrónicos utilizando herramientas de inteligencia artificial. Instito Politecnico Nacional IPN-CIDETEC, México (2016)

4. Dillip Kumar, M.: Application of firefly algorithm for design optimization of a shell and tube heat exchanger from economic point of view. International Journal of Thermal Sciences, Vol. 1, pp. 228-238 (2016)

5. Galeano Ureña, C.H., Duque Daza, C.A., Garzon Alvarado, D.A.: Aplicación de diseño óptimo dimensional a la síntesis de posición y velocidad en mecanismos de cuatro barras. Revista Facultad de Ingenieria Universidad de Antioquia, No. 47, pp. 129-144 (2008) 
Maria Bárbara Calva Yáñez, Paola Andrea Niño Suárez, Jorge Alexander Aponte Rodríguez, et al.

6. Valentin, E.S., Solano, P.A., Bautista, C.P., Rueda Melendez, J.M., Portilla Flores, E.A.: Diseño óptimo para transmisión de fuerza en un efector final. Research in Computing Science, No. 91, pp. 117-130 (2015)

7. Price, K.V.: An introduction to differential evolution. U.K. Maindenhead: Mc Graw - Hill (1999)

8. Deb, K.: An effcient constraint handling method for genetic algorithms. Computer Methods in Applied Mechanics and Engineering, Vol. 186, pp. 311-338 (2000)

9. Shruti Goel, V.K.P.: Performance Evaluation of a New Modified Firefly. 3rd International Conference on Reliability, Infocom Technologies and Optimization (ICRITO) (Trends and Future Directions), Noida (2014)

10. Michalewicz, Z., Schoenauer, M., Koziel, S.: Evolutionary Algorithms, Homomorphous Mappings, and Constrained Parameter Optimization. Evolutionary Computation, Vol. 7, No. 1, pp. 19-44 (1999)

11. Yang, X.-S.: Firefly Algorithm, Levy Flights and Global Optimization. Research and Development in Intelligent System, Vol. 26, pp. 209-218 (2010) 